\title{
Die Sippen-Tabus der Kinga ${ }^{1}$ \\ THILO C. SCHADEBERG
}

1. Die Kinga bewohnen den nördlichen Teil der Livingstone-Berge zwischen $9^{0} 10^{\prime}$ und $9^{0} 45^{\prime} \mathrm{s}$. Br. und $33^{\circ} 55^{\prime}$ und $34^{0} 30^{\prime}$ ö. L. Die hohe Lage zwischen $1500 \mathrm{~m}$ und $2000 \mathrm{~m}$ und die lange Regenzeit von Dezember bis April begünstigen einen verhältnismäßig intensiven Ackerbau. Die Kinga zählen heute zwischen 50000 und 100000 Menschen. Sie sprechen eine eigene Bantusprache; dazu ist die Kenntnis der ostafrikanischen Verkehrssprache, des Swahili, unter ihnen weit verbreitet.

Im Jahre 1894 gründeten die Berliner Missionare Schumann und Wolff die erste Missionstation im Kinga-Land ${ }^{2}$. Seither hat das Wirken verschiedener lutherischer Missionen unter den Kinga großen Einfluß ausgeübt. Heute sind die sechs lutherischen Kinga-Gemeinden Teil der Südlichen Synode der Evangelisch-Lutherischen Kirche von Tanzania.

Von den Nachbarn der Kinga seien hier nur die Nyakyusa aus dem Gebiet nördlich des Malawi-Sees genannt, die - auch unter der Bezeichnung Konde - verschiedentlich in der ethnologischen und Missions-Literatur dargestellt worden sind.

2. Jeder Kinga ist durch einen seiner Namen als Mitglied einer größeren Gruppe ausgewiesen. Die Mitgliedschaft dieser Gruppen wird partrilineal vererbt und ist unaufgebbar. Alle Mitglieder einer Gruppe sind unterein. ander blutsverwandt. Da manche Gruppen sehr ausgedehnt sind, ist der exakte genealogische Zusammenhang nicht immer aufweisbar. Murdock

1 Von Oktober 1966 bis Juni 1967 hielt ich mich in Tanzania auf, um Material für eine Dissertation über die Sprache der Kinga zu sammeln. Dieses Projelkt wurde durch die Unterstützung der Klopstock-Stiftung, Hamburg, des Deutschen Akademischen Austauschdienstes und der Philipps-Universität, Marburg, ermöglicht.

Zum Verständnis der Schreibung der Kinga-Laute mögen noch folgende Hinweise dienen: Die sieben Vokale der Kingasprache bezeichne ich mit a e $\underline{i} i$ $o \underline{u} u$. Dabei stehen $\underline{i}$ und $\underline{u}$ in bezug auf ihren Öffnungsgrad zwischen $e$ und $i$ bzw. $o$ und $u$. Die Akzente über den Vokalbuchstaben bezeichnen die Töne: ' (Tiefton); ' (Hochton); ^ (Fallton). In Eigennamen bleibt der Ton unbezeichnet. $k$ ist, wenn es nicht in einer Nasalverbindung steht, wie der velare Frikativlaut im deutschen Wort ,,acht" zu sprechen.

2 Vgl. M. Ermerar, Berlinor Mission im Njaßa-Lande (Deutsch-Ostafrika), Berlin 1897, S. 94. 
bezeichnet solche Gruppen mit $s i b^{3}$. Solche Gruppen können natürlich keine lokalen Einheiten darstellen, da ja bei der Eheschließung und Neugründung eines Haushaltes die väterlicherseits ererbte Sippenzugehörigkeit beibehalten wird. Die Sippen sind - zumindest heute - nicht exogam. Daher können zwischen zwei Mitgliedern einer Sippe auch affinale Beziehungen (durch Anheirat) bestehen. Konstituierend bleibt für die gemeinsame Sippenzugehörigkeit aber die Blutsverwandtschaft.

3. Folgende Sippennamen konnte ich notieren:

$\begin{array}{lll}\text { Fungo } & \text { Mbilingi } & \text { Nyaluke } \\ \text { Ilomo } & \text { Mbogela } & \text { jwavi } \\ \text { Kúsiluka } & \text { Mbwilo } & \text { Pela } \\ \text { Kyando } & \text { Mwamasangola } & \text { Sanga } \\ \text { Kyavula } & \text { Mwangoma } & \text { Sigala } \\ \text { Ligate } & \text { Ndelwa } & \text { Sovela } \\ \text { Luvanda } & \text { Ngajilo } & \text { Swalo } \\ \text { Lwila } & \text { Ngulwa } & \text { Tete } \\ \text { Mahenge } & \text { Nsemwa } & \text { Tweve }\end{array}$

4. Die Bedeutungen dieser Sippennamen sind den Kinga heute nicht bekannt. Nur für den Namen „Sanga” erhielt ich eine Deutung: „Sie kamen aus der Gegend um Ukwama. Sie zogen in das Tal von Bulongwa. Sie sagten immerzu: sanga! sanga!, d. h. Einsammeln! Zusammenbringen!" (Zur Erläuterung: Die Sippe der Sanga erorberte einmal die Vormachtstellung über den größten Teil der Kinga. Sie stellt bis heute die Häuptlinge. Vgl. auch ịli-sângo ,Steuer, Abgabe”.)

Es ist allerdings möglich, einen Teil dieser Namen zumindest annähernd zu übersetzen. $\mathrm{Ob}$ auf diese Weise auch die historische Bedeutung erkannt ist, muß bezweifelt werden.

Einige solche Übersetzungsversuche seien hier angeführt:

Ilomo: $i$ - nicht sicher identifiziertes Element, das häufig in Eigennamen auftritt

undòmo (Stamm -lòmo) „Mund, Lippe"

Kyavula: ,etwas zum Sagen"

kya. Konnektiv Kl. 7

-vứla ,sagen"

Luvanda: lu- Präfix K1. 11

umbànda (Stamm -vdnda), ,Landsmann, Bürger"

iki-vânda ,Maisfladen”

a Gmoram Pempr Murdock, Social Structure, New York 1949, S. 47 : „, When the members of a consanguineal kin group acknowledge a traditional bond of common descent in the paternal or maternal line, but are unable always to trace the actual genealogical connections between individuals, the group is called a sib." 
Lwila: -lwilla ,kämpfen, appl. (für oder gegen)"

Mahenge: $m a$ - Präfix Kl. 6

-hênga ,mähen"

uvur-hènge "gemähtes Stück Land"

Ndelwa: „der Aufgezogene"

-lella ,,(Kinder) aufziehen"

$-w$ - Passiv-Erweiterung

Ngulwa: ,der Gekaufte"

-gùla „kaufen"

$-w$ - Passiv-Erweiterung

Pela: -péla (appl. zu -pà ,geben”) „etwas zum Trinken in die Hand gießen"

-pèla 1. ,erschaffen"

2. ",bereuen"

Sigala : -sigàla ,"übrig bleiben"

Swalo: -swâla ,,anziehen, tragen (Kleidung)"

-o Nominalsuffix

In keinem Falle kann ich einen Zusammenhang zwischen diesen Sippennamen und den einzelnen Sippen-Tabus der Kịnga erkennen.

5. Diese Sippen stehen sich nicht gleichwertig gegenüber. Viele sind so klein und auf ein so enges Gebiet begrenzt, da $B$ der genealogische Zusammenhang vielleicht noch klar durchschaubar ist. Nach Murdock sollten diese Gruppen besser nicht sibs, sondern lineages genannt werden ${ }^{4}$. Solche „Klein-Sippen” mögen die Reste ehemals größerer Sippen darstellen, ihr gemeinsamer Vorfahr mag erst vor wenigen Generationen aus dem Gebiet eines Nachbarstammes eingewandert sein, sicher entstehen sie aber auch durch Abspaltung von den großen, alteingesessenen Sippen. Dieser letztere Vorgang läßt sich auch heute beobachten: Die oben erwähnte „Häuptlingssippe” der Sanga ist die zahlreichste und am weitesten verbreitete Sippe. Lokale Untergruppen der Sanga sind die Nkwama, Lupondo, Mwihoneke u. a. m. Nkwama ist ein rom Órtsnamen der Herkunft (, Unkwama") abgeleiteter „Familienname”. Lupondo war ebenfalls eine Ortsbezeichnung; heute dient er zur Bezeichnung einer lineage (der Sanga-Sippe), deren Vorfahr sich an diesem Ort niederließ. Mwihoneke schließlich war der Eigenname eines Mannes der Sanga-Sippe, der an einem neuen Ort eine neue lineage

4 G. P. MURDock, op. cit., S. 46: „A consanguineal kin group produced by either rule of unilinear descent is technically known as a lineage when it includes only persons who can actually trace their common relationship through a specific series of remembered genealogical links in the prevailing line of descent." 
gründete. Sein Name diente dann zur Bezeichnung dieses Ortes und heute als Familienname für seine patrilinealen Nachkommen.

Für die meisten Sippen kann ein Kerngebiet oder Ausbreitungszentrum angegeben werden. Die mündliche Tradition kann die Geschichte der Sanga bis zu ihrer Einwanderung aus dem benachbarten Bena-Land vor mehreren hundert Jahren zurückverfolgen. Sie siedelten zuerst bei Ukwama, von dort breiteten sie sich und ihre Macht über das ganze Kinga-Land aus. In dem im westlichen Kinga-Land gelegenen MahanziGebiet ist überliefert, daß die Sippe der Kyando dort schon vor der Ankunft der Sanga ansässig war und auch die Häuptlinge stellte. Ähnlich verhält es sich mit der Sippe der Lwịla im Südteil des Kịnga-Landes. Leider ist uns über die Geschichte der Kinga insgesamt fast nichts bekannt. Ist es ein Volk, das aus vielen kleinen Stämmen und Grüppchen zusammengewachsen ist? Oder sind die Kinga ein Volk, das schon seit sehr langer Zeit in diesem Bergland wohnt und nur Akkumulator für andere, Zuflucht heischende Gruppen wurde? Die Lösung dieser Frage steht noch aus und kann auch hier - so nützlich es für eine Deutung der Sippen-Tabus wäre - nicht geleistet werden. Jedoch deuten die mündlichen Überlieferungen der einzelnen Sippen - soweit sie mir bekannt geworden sind - mehr auf die erste der beiden Möglichkeiten hin, da sie meist auf einen Ursprung außerhalb des Kinga-Landes verweisen oder sich selbst (im Falle der Kyando-Sippe) den einwandernden "Kinga” (gemeint sind wohl die Sanga-Leute) gegenüberstellen.

6. Das Kinga-Wort für „Meidung, Tabu” heißt umwiko. Zur allgemeinen Definition diene das Zitat: ,Tabu, in short, is to be understood as meaning ,unlucky to meddle with' rather than forbidden by edict human or divine."

Neben allgemein gültigen Tabus oder Meidungen gibt es bei den Kịnga auch solche, die jeweils einer bestimmten Sippe eigen sind. Die folgenden konnte ich notieren:

Fungo: inzùilu ,großes rattenartiges Tier mit langem Schwanz"

Kyavula: isùde „Hase" imbávalla „Buschbock"

Luvanda: iside „Hase”

Sanga: issìde „Hase" uvwânàsýàle „Niere”

Erklärung: Der Genuß all dieser ,Fleischsorten" ist zu meiden. Lwila: in ùmbàtsi „eine Art Ratte”

Erklärung: Bricht dieses Tier in einen Speicher ein, wird alles darin tabu.

5 R. R. MarRetT in Encyclopaedia of Religion and Ethios, Hrsg. James Hastings, Edinburgh 1954, Vol. 12, S. 183 (Stichwort „Tabu”). 
îlivyâvya ,,eine Art Blätter”

Erklärung: In diese Blätter darf man keine Speisen einwickeln

Nyalukke: ijibùdillwè nụlùma ,,(Tier) was vom Leoparden getötet wurde"

Erklärung: Alles Fleisch von einem Tier, das der Leopard getötet hat, ist tabu.

நwavi: ikibùdu ,etwas Totes" (sonst ungebräuchliches Wort)

Erklärung: Fleisch von einem Tier, dessen Kopf man nicht gesehen hat, ist tabu.

Sovela: uvvuvalìndnye ,das Aufeinander-gestellt-sein" (sonst ungebräuchliches Wort)

Erklärung: In einem Topf dürfen auch bei der Zubereitung nicht zwei verschiedene Speisen übereinander liegen.

Ilomo: ilkyàle „Gebärhütte”

Erklärung: Nur die Gebärende darf diese Hütte betreten. Von der Geburt an darf einen Monat lang niemand Mutter und Kind sehen.

$i k i b d s u$,das Blinde"

Erklärung: Blind geborene Tiere dürfen so lange nicht angeschaut werden, bis sie sehen.

inàta „,der Ring, der beim Tragen schwerer Lasten auf dem Kopf untergelegt wird"

Erklärung: Man darf eine Frau nicht grüßen, solange sie den Tragring auf dem Kopf hat. Man darf nicht an einem Tisch essen, auf dem der Tragring liegt.

Mahenge: wie Ilomo, s. o.

Ngajilo: imbwà „Hund"

Erklärung: Man darf nicht hinschauen, wo eine Hündin ihr Lager mit ihren Jungen hat.

Tweve: ihiùtútu ,ein großer schwarzer Vogel”

Erklärung: Man darf ihn nicht ansehen oder an ihm vorübergehen, wenn er auf dem Wege sitzt. Man kann dann mit abgewandtem Gesicht folgendes Verschen singen: túllùtè kânnu, twị̂vànyà Twêve „Wir wollen hier vorbeigehen, wir Tweve-Leute".

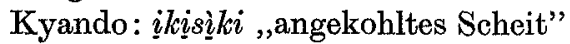

Erklärung: Ein halb verbranntes Stück Holz darf nicht wieder zum Feuern verwendet werden. Man darf ein angekohltes Scheit auch nicht zum Stochern im Feuer verwenden.

7. Für diese Tabus schlage ich folgende Einteilung nach der Art der Meidung vor:

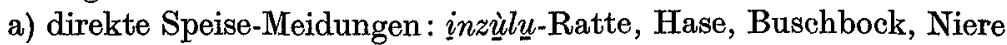

b) indirekte Speise-Meidungen: bestimmte andere Dinge tabuisieren Speisen: îùmbàtsi-Ratte, ilivyâvya-Blätter, das Töten durch den Leopar- 
den, das Fehlen oder Nicht-gesehen-haben des Kopfes, das Utbereinanderliegen von Speisen in einem Topf, die Nachbarschaft des inàta-Ringes auf dem Tisch

c) Sicht-Meidungen: die Gebärhütte und Mutter und Kind im ersten Monat nach der Geburt, noch blinde junge Tiere, (noch blinde?) junge Hunde, der ihìtútu-Vogel

d) Gebrauchs-Meidung: das angekohlte Scheit

Die Zuordnung der ī $y$ dta-Meidung ist nicht eindeutig. Wegen der Kraft dieses Tragringes, das Grüßen zu verhindern, könnte man hier vielleicht eine spezielle Form der Sicht-Meidung vermuten. ${ }^{6}$

8. Ich habe nicht beobachten können, daß diese Meidungen heute noch bewußt eingehalten würden. Ein direktes Verbot durch die Missionare dürfte kaum die Ursache sein, da mir von Christen versichert wurde, daß es nicht unchristlich sei, wenn jemand eine solche Meidung beachten wolle. Wie groß der Abstand zu diesen Dingen heute ist, mag an

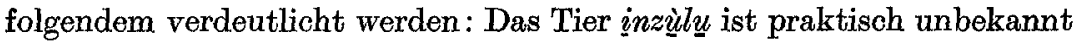
und die Schilderungen seines Aussehens differieren dementsprechend beträchtlich. Ein anderes Beispiel: das Blatt illivyâvya wird sowieso von niemandem zum Einwickeln von Speisen verwendet. Andere Meidungen wiederum können zu allgemeinen Verhaltens- oder ,Anstands"-Regeln werden, z. B. daß man nicht von einem Tisch ißt, auf dem der inàta-Ring liegt.

Der hier aufgezeigte Abstand erklärt dann wohl auch die lückenhafte und sicher teilweise auch ungenaue Aufnahme der Data. Ich halte es beispielsweise für wahrscheinlich, daß die beiden angeführten Meidungen ikibdsu , das Blinde" und imbwà , der Hund" eigentlich identisch sind. In diesem Fall hätte sich mein Informant für die Meidung imbwd eben nur noch an den zwar häufigsten, aber doch speziellen Fall ,junge Hunde" erinnern können, aber nicht mehr an das allgemeinere Tabu ,blind geborene junge Tiere".

Bei dieser Lage der Dinge ist es auch nicht verwunderlich, daß ich wenige Auskünfte darüber erhalten konnte, was denn - nach Meinung „der Alten" - geschähe, wenn eine Meidung durchbrochen würde. Die

${ }^{6}$ Vgl. auch den Katalog der Meidungen in Leviticus, Kap. 11. Hier finden sich direkte und indirekte Speise-Meidungen und Kontakt-Meidungen. Sie sind aber eng ineinander verflochten und beziehen sich oft auf gleiche Objekte, vgl. die Bestimmungen über das Aas in V, 11, 24, 27-28, 31-33, 35-40. Als Beispiele seien drei Verse in der Luther-Übersetzung zitiert: V. 11: daß ihr von ihrem Fleisch nicht esset und vor ihrem Aas euch scheuet. (direkte Speisemeidung) V. 24: . . Wer solches Aas anrührt, der wird unrein sein bis auf den Abend. (Kontaktmeidung) V. 33: Allerlei irdenes Gefäß, wo solcher Aase eines hineinfällt, wird alles unrein, was darin ist; und sollts zerbrechen (doppelt indirekte Speisemeidung). 
Vorstellungen gehen meist nicht über das hinaus, was Ernst Dammann allgemein sagt: ,,.. Verbote bestehen, die vor unangenehmen Auswirkungen der Macht schützen sollen."7

Eine Besonderheit ist hier allerdings mitzuteilen: Eine Mißachtung der Meidung ikibòsu ,das Blinde” hat nicht für den Täter unangenehme Folgen, sondern führt zum Tod der betroffenen Tiere. Im Normalfall ist der Täter auch nicht deren Besitzer oder Sippengenosse des Besitzers, so daB der Verlust ihn nicht einmal indirekt trifft. Hier wird ein allgemeiner Wesenszug des Tabus sichtbar. ,,Verletzung des tabus hat keine Strafe zur Folge, sondern ein automatisches Reagieren der Macht." "Das Tabu ist streng zu unterscheiden von dem Komplex, der durch die Begriffe Verbot - Vergehen - Sühne oder Strafe umrissen werden kann. „Der Mechanismus des Tabu ist indessen immer derselbe: gewisse Sachen, Personen oder Gebiete haben an einem ganz anderen ontologischen Bereich teil, und notwendig bedeutet ein Kontakt mit ihnen das Durchbrechen einer ontologischen Ebene (rupture de niveau ontologique), was verhängnisvoll werden kann."9

9. Die Existenz von Sippentabus ist nicht nur bei den Kinga und ihren Nachbarn anzutreffen. Sie wird auch aus so unterschiedlichen und weit auseinander liegenden Gegenden Afrikas berichtet wie von den Herero ${ }^{10}$, den Nuba ${ }^{11}$ und den Edo aus Nigeria ${ }^{12}$. Eine vergleichende Betrachtung liegt aber nicht im Bereich dieser Arbeit.

10. „Bekanntlich gehört es zu den schwersten Aufgaben der Religionswissenschaft, für die unzähligen Tabuverbote, deren Übertretung von üblen Folgen ist, irgendeinen Grund anzugeben."13 Ernst Dammann umreißt die theoretisch möglichen Deutungen wie folgt: „,Die Gründe für die Speisetabus dürften verschieden sein. Totemistische Vorstellungen, Verbundenheit mit Tier und Pflanze, mythologische Anschauungen ... oder Analogievorstellungen ... mögen bei dem Entstehen der Meidungen mitgewirkt haben."14 Darüber hinaus gibt es noch die Möglichkeiten der psychologischen Deutung. Offen bleibt aber die Frage: Wie ordne ich einer bestimmten Meidung eine bestimmte Deutung zu ?

7 Ernst Dammann, Die Religionen Afrikas, Stuttgart 1963, S. 94.

${ }^{8}$ G. VAN DER LeEUW, Phänomenologie der Religion, Tübingen 1956, S. 31.

- Mrrcea Eliade, Die Religionen und das Heilige, Salzburg 1954, S. 40.

10 E. DammanN, op. cit., S. 41.

11 S. F. Nadex, Dual Descent in the Nuba Hills, in: African Systems of Kinship and Marriage, hrsg. von A. R. Radolmme-Brown and DaryLL Forde, S. 339 340.

12 G. P. MURDOCK, op. cit., S. 48.

${ }^{13}$ Konrad Theodor Preuss, Religiöses Recht und Königtum in Amerika; in: Zeitschrift für vergleichende Rechtswissenschaft, Bd. 50, 1936, S. 269.

14 E. Dammann, op. cit., S. 96. 
Von den Kinga selbst konnte ich keine Informationen über den Ursprung der Sippentabus bekommen. Einem Deutungsversuch von außen müßte erst einmal eine Hypothese darüber vorausgehen, ob diese Sippentabus ein System mit einem Ursprung darstellen oder als ein Konglomerat von Tabus verschiedenartigster Herkunft aufzufassen sind. Es ist dies die gleiche Frage wie die oben gestellte nach dem Werden des Kinga-Volkes überhaupt. Sodann müßte geklärt werden, warum manche Sippen nur eine eigene Meidung haben, andere aber mehrere. Auch die Wiederkehr der gleichen Meidung für mehrere Sippen verlangt eine Deutung.

Auf diesen Gebieten können die Aufzeichnung und die Bearbeitung der mündlich überlieferten Traditionen der Kinga und ihrer Nachbarvölker noch weiterführende Ergebnisse bringen. Aber der Ursprung und die Bedeutung der Sippentabus werden den Kịnga selbst immer dunkler und wohl auch uns nie völlig klar werden. 